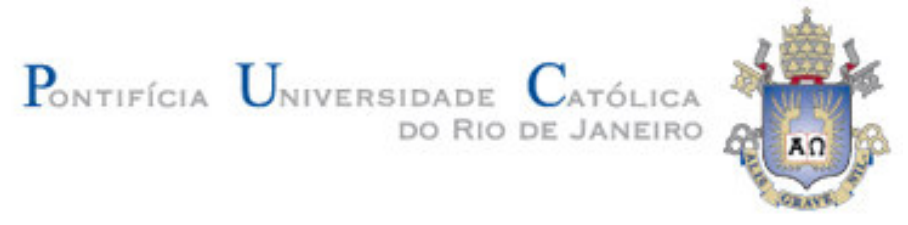

Paula Tavares da Silva

\title{
Medidas de Desempenho Econômico e Geração de Valor: \\ O Caso das Empresas Brasileiras de Capital Aberto
}

\author{
Dissertação de Mestrado
}

Dissertação apresentada como requisito parcial para obtenção do grau de Mestre pelo Programa de PósGraduação em Engenharia de Produção do Departamento de Engenharia Industrial da PUC-Rio.

Orientador: Prof. Carlos Patrício Samanez

Rio de Janeiro

Agosto de 2010 


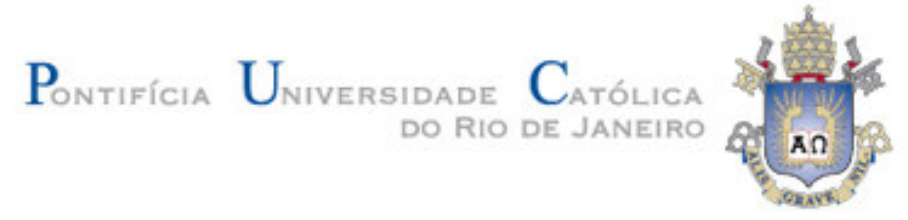

Paula Tavares da Silva

Medidas de Desempenho Econômico e Geração de Valor:

O Caso das Empresas Brasileiras de Capital Aberto

Dissertação apresentada como requisito parcial para obtenção do grau de Mestre pelo Programa de PósGraduação em Engenharia de Produção do Departamento de Engenharia Industrial da PUC-Rio.

Prof. Carlos Patrício Samanez Orientador Departamento de Engenharia Industrial - PUC-Rio

Prof. José Paulo Teixeira Departamento de Engenharia Industrial - PUC-Rio

Prof. Antônio Carlos Gonçalves Universidade Federal Rural do Rio de Janeiro - UFFRJ

Prof. José Eugênio Leal Coordenador Setorial do Centro Técnico Científico - PUC-Rio 
Todos os direitos reservados. É proibida a reprodução total ou parcial do trabalho sem autorização da universidade, da autora e do orientador.

\section{Paula Tavares da Silva}

Graduou-se em Engenharia de Sistemas e Computação na UERJ (Universidade do Estado do Rio de Janeiro) em 1997 e em Medicina na Uni-Rio (Universidade do Rio de Janeiro) em 2003.

Ficha Catalográfica

CDD: 658.5 


\title{
Agradecimentos
}

\author{
Ao meu orientador Professor Carlos Patrício Samanez pelo acompanhamento na \\ elaboração deste trabalho. \\ Ao CNPq e à PUC-Rio, pelos auxílios concedidos, sem os quais este trabalho não \\ poderia ter sido realizado.
}

Aos professores que participaram da Comissão examinadora.

A todos os professores do Departamento pelos ensinamentos e pela ajuda.

E, sobretudo, à minha família, Joana e Maria da Conceição, exemplos maiores de força, perseverança e dedicação. 


\section{Resumo}

Da Silva, Paula Tavares; Samanez, Carlos Patrício. Medidas de Desempenho Econômico e Geração de Valor: o caso das empresas brasileiras de capital aberto. Rio de Janeiro, 2010. 197p. Dissertação de Mestrado - Departamento de Engenharia Industrial, Pontifícia Universidade Católica do Rio de Janeiro.

A presente dissertação tem por objetivo a análise empírica do desempenho de uma amostra representativa das empresas brasileiras e de seus setores no período compreendido entre 1999 e 2008, através da medida de desempenho que tenha uma maior relação com o retorno dos ativos no mercado de capitais. A fim de se alcançar este objetivo, fez-se necessário obter valores de medidas de desempenho tradicionais das empresas, tais como o ROI, ROA, ROE e LPA e de medidas baseadas na criação de valor para o acionista, como o EVA (Valor Econômico Adicionado), para em seguida, correlacioná-los à medida de desempenho externa, o MVA (Valor de Mercado Agregado), tida como a que melhor reflete o retorno dos ativos, pois além de considerar o desempenho corrente da empresa, leva em conta as expectativas futuras do mercado em relação à mesma. Para este estudo, foram analisadas 47 empresas com capital aberto na BOVESPA, distribuídas em 12 setores da economia brasileira. A mais alta correlação obtida foi entre o EVA e o MVA da empresas, corroborando com a afirmação da empresa detentora dos direitos sobre o EVA, Stern Stewart \& Co., de que o EVA é uma medida superior de desempenho de empresas. Usando o EVA como métrica de desempenho, foi observado que somente cinco das 47 empresas avaliadas construíram valor para o acionista ao longo da década, que os setores de petróleo e de mineração foram os que mais criaram riqueza para o país, e que o período associado ao governo Lula foi marcado por um acentuado aumento das expectativas de crescimento de EVAs futuros.

\section{Palavras-chave}

EVA; MVA; custo de capital; lucro econômico; lucro contábil. 


\section{Abstract}

Da Silva, Paula Tavares; Samanez, Carlos Patrício (Advisor). Measures of Economic Performance and Value Creation: the case of Brazilian companies. Rio de Janeiro, 2010. 197p. MSc. Dissertation Departamento de Engenharia Industrial, Pontifícia Universidade Católica do Rio de Janeiro.

This essay aims the empirical analysis of the performance of a representative sample of brazilian companies and their sectors over the period 1999-2008, through the measure of performance that has greater relation to the return of assets in the capital market. In order to achieve this goal, it was necessary to obtain values of traditional performance measures, such as ROI, ROA, ROE and EPS, as well as measures based on the creation of shareholder value, such as EVA (Economic Value Added), to, afterwards, correlate them with the external performance measure, MVA (Market Value Added), regarded as the one that best reflects the return of assets, because it considers the company's current performance and takes into account future market expectations. This essay was based on a sample of 47 companies traded on BOVESPA, distributed in 12 sectors of the Brazilian economy. The highest correlation obtained was between EVA and MVA corroborating with Stern Stewart \& Co's statement that EVA is a superior measure of business performance. When using EVA as the performance measure, it was noted that only five of 47 companies evaluated built shareholder value over the decade, that the petroleum and mining sectors were the ones that have created more wealth for the country, and that the period associated with the Lula's Government was marked by a sharp increase in expectations of future growth of EVAs.

\section{Keywords}

EVA; MVA; economic value added; market value added; cost of capital; economic profits; accounting profits; earnings. 


\section{Sumário}

$\begin{array}{ll}\text { 1. Introdução } & 14\end{array}$

2. Fundamentação Teórica 18

2.1. Modelos contábil e econômico de avaliação de uma empresa 18

2.2. Medidas de desempenho tradicionais 23

2.2.1. Lucro Líquido 23

2.2.2. EPS ou Lucro por ação 23

2.2.3. Índice valor de mercado/valor patrimonial ou Market to book ratio 24

2.2.4. Retorno sobre o Ativo (ROA) 24

2.2.5. Retorno sobre o Investimento ou sobre o Capital Investido (ROI) 25

2.2.6. Retorno sobre o Patrimônio Liquido (ROE) 26

2.3. Medidas de desempenho baseadas em Valor 27

2.3.1. Fluxo de Caixa Descontado (FCD) 28

2.3.2. Método de Valor Econômico Agregado 31

2.3.2.1. $\mathrm{EVA}^{\circledR}$

2.3.2.1.1. A Origem 32

2.3.2.1.2. O Conceito 33

2.3.2.1.3. Usos do EVA 36

2.3.2.1.4. Prós e Contras 39

2.3.2.2. $M V A^{\circledR}$ (Market Value Added) 42

2.3.3. Relação entre EVA e MVA

2.3.4. Relação do EVA com o retorno dos ativos 46

2.3.5. Ferramentas utilizadas no cálculo do EVA 50

2.3.5.1. Ajustes Contábeis $\quad 50$

2.3.5.2. Cálculo do custo de capital 53

2.3.5.2.1. Cálculo do custo do capital de terceiros 55

2.3.5.2.2. Cálculo do custo do capital próprio 56 
3. Metodologia $\quad 59$

3.1. Amostra 59

3.2. Cálculos do NOPAT, capital investido e custo do capital 62

3.3. Relação entre índices 68

4. Análise dos Resultados $\quad 71$

4.1. EVA como indicador de desempenho superior 71

4.2. Relação entre $E V A^{\circledR}{\text { e } M V A^{\circledR}}^{\circledR} 76$

4.3. Evolução dos indicadores médios (EVA e MVA) na década 77

4.4. Desempenho relativo dos setores da economia 81

4.5. Avaliação dos setores em qüinqüênios 84

4.6. Avaliação das empresas dentro dos setores 85

4.6.1. Comércio 85

4.6.2. Eletroeletrônico

4.6.3. Energia Elétrica $\quad 85$

4.6.4. Mineração 86

4.6.5. Papel e Celulose $\quad 86$

4.6.6. Petróleo 86

4.6.7. Química $\quad 86$

$\begin{array}{lr}\text { 4.6.8. Siderurgia } & 87\end{array}$

4.6.9. Telecomunicações $\quad 87$

4.6.10. Têxtil $\quad 87$

4.6.11. Transportes $\quad 88$

4.6.12. Veículos e Peças $\quad 88$

5. Conclusão $\quad 89$

6. Referências 92

7. Anexol 95

8. Anexo II 96 


\section{Lista de Tabelas}

Tabela 2.1. Comparação entre Métricas de Desempenho 22

Tabela 2.2. Ajustes contábeis típicos feitos pela consultoria Stern \& Stewart 52

$\begin{array}{ll}\text { Tabela 3.1. Empresas por patrimônio líquido } & 60\end{array}$

Tabela 3.2. Cálculo do NOPAT 62

Tabela 3.3. Balanço da ALL em dezembro de $1998 \quad 64$

Tabela 3.4. Exemplo de cálculo do capital investido de uma empresa 64

Tabela 3.5. Taxa Selic $\quad 66$

Tabela 4.1. Correlação entre medidas de desempenho da amostra entre 1999 e 2008

72

Tabela 4.2. Correlação entre medidas de desempenho da amostra entre 1999 e 2003

72

Tabela 4.3. Correlação entre medidas de desempenho da amostra entre 2004 e 2008

Tabela 4.4. Correlação entre EVA e MVA

Tabela 4.5. Resultado das regressões simples entre MVA e EVA, ROE e EVA padronizado

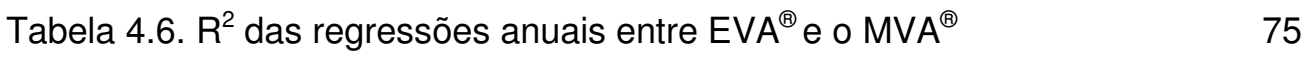

Tabela 4.7. Características da amostra 76

Tabela 4.8. Correlação entre medidas de desempenho da amostra entre 1999 e 2008, sem a empresa Guararapes.

Tabela 4.9. Correlação anual entre EVA ${ }^{\circledR}{\text { e } M V A^{\circledR}}^{\circledR}$ da amostra, excluindo a Guararapes.

Tabela 4.10. Resultado das regressões entre MVAp e EVAp, ROE, ROI, $\quad 80$ ROA e LPA; e entre MVA e EVA (sem a Guararapes).

Tabela 4.11. EVA médio do setor de comércio 85

Tabela 4.12. EVA médio do setor eletroeletrônico 85

Tabela 4.13. EVA médio do setor de energia elétrica 86

Tabela 4.14. EVA médio do setor de papel e celulose 86

$\begin{array}{ll}\text { Tabela 4.15. EVA médio do setor químico } & 87\end{array}$

Tabela 4.16. EVA médio do setor siderúrgico 87 
Tabela 4.17. EVA médio do setor de telecomunicações

Tabela 4.18. EVA médio do setor têxtil

88

Tabela 4.19. EVA médio do setor de veículos e peças

88

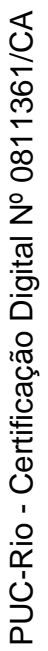




\section{Lista de Figuras}

Figura 2.1. Cálculo do Lucro Econômico 20

Figura 2.2. Cálculo do Fluxo de Caixa Livre 28

Figura 2.3. Cálculo do EVA. $\quad 34$

Figura 2.4. Valor prêmio e valor descontado. 44

Figura 2.5. Espectro de EVAs $\quad 51$

Figura 2.6. Trade-off Risco-retorno $\quad 57$

Figura 4.1. Evolução dos indicadores médios entre 1999 e 2008

Figura 4.2. Evolução dos indicadores médios entre 1999 e 2008 $\begin{array}{ll}\text { sem a Guararapes } & 79\end{array}$

Figura 4.3. Evolução do EVA entre 1999 e 2008 por setor da economia brasileira

82

Figura 4.4. Evolução do MVA entre 1999 e 2008 por setor da economia brasileira 


\section{Lista de Abreviatura e Símbolos}

\begin{tabular}{|c|c|}
\hline ANBID & Associação Nacional de Bancos de Investimento \\
\hline BNDES & Banco Nacional de Desenvolvimento Econômico e Social \\
\hline BOVESPA & Bolsa de Valores de São Paulo \\
\hline CAPM & Capital Asset Pricing Model \\
\hline CMPC & Custo Médio Ponderado de Capital \\
\hline CEO & Chief Executive Officer \\
\hline CFROI & Cash Flow Return on Investment \\
\hline CSSL & Contribuição Social sobre o Lucro \\
\hline CVA & Cash Value Added \\
\hline CVM & Comissão de Valores Mobiliários \\
\hline DPS & Dividends per Share \\
\hline DRE & Demonstração do Resultado do Exercício \\
\hline EBIT & Earns Before Interest and Taxes \\
\hline EBITDA & Earns Before Interest, Taxes, Depreciation and Amortization \\
\hline EPS & Earns per share ou lucro por ação \\
\hline EVA & Economic Value Added ou Valor Econômico Adicionado \\
\hline FCD & Fluxo de Caixa Descontado \\
\hline $\mathrm{FCL}$ & Fluxo de Caixa Livre \\
\hline $\mathrm{FCO}$ & Fluxo de Caixa Operacional \\
\hline FIFO & First In First Out \\
\hline g & Taxa de Crescimento de Fluxo \\
\hline GAAP & Generally Accepted Accounting Principles \\
\hline $\mathrm{K}$ & Custo de Capital \\
\hline $\mathrm{K}_{\mathrm{d}}$ & Custo do Capital de Terceiros \\
\hline $\mathrm{K}_{\mathrm{p}}$ & Custo do Capital Próprio \\
\hline
\end{tabular}




\begin{tabular}{|c|c|}
\hline LAJIDA & Lucro Antes de Juros, Impostos, Depreciação e Amortizacão \\
\hline LIFO & Last In First Out \\
\hline LPA & Lucro por Ação \\
\hline MVA & Market Value Added ou Valor de Mercado Agregado \\
\hline NOPAT & Net Operational Profits After Taxes ou resultado operacional liquid \\
\hline $\begin{array}{l}\text { NOPBT } \\
\text { bruto }\end{array}$ & Net Operacional Profit Before Taxes ou resultado operacional \\
\hline NPV & Net Present Value \\
\hline $\mathrm{P} / \mathrm{L}$ & Razão Preço/Lucro \\
\hline$P \& D$ & Pesquisa e Desenvolvimento \\
\hline $\mathrm{Rf}$ & Renda Fixa \\
\hline $\mathrm{Ri}$ & Retorno esperado do ativo i \\
\hline $\mathrm{Rm}$ & Retorno esperado da carteira de mercado \\
\hline ROA & Return on Assets Retorno sobre os Ativos \\
\hline ROE & Return on Equity ou Retorno sobre o Patrimônio Líquido \\
\hline $\mathrm{ROI}$ & Return on Investments ou Retorno sobre os Investimentos \\
\hline SVA & Shareholder Value Analysis \\
\hline $\mathrm{T}$ & Tributos \\
\hline TBR & Total Business Return \\
\hline TRS & Total Returns to Shareholders \\
\hline VBM & Value-based Management \\
\hline VEA & Valor Econômico Agregado \\
\hline VCF & Valor de Crescimento Futuro \\
\hline VOC & Valor das Operações Correntes \\
\hline VPL & Valor Presente Líquido \\
\hline WACC & Weighted Average Cost of Capital \\
\hline
\end{tabular}

